\title{
Video Article \\ Microvascular Decompression: Salient Surgical Principles and Technical Nuances
}

\author{
Jonathan Forbes ${ }^{1}$, Calvin Cooper ${ }^{*}$, Walter Jermakowicz ${ }^{2}$, Joseph Neimat ${ }^{1}$, Peter Konrad ${ }^{* 1}$ \\ ${ }^{1}$ Department of Neurosurgery, Vanderbilt University Medical Center \\ ${ }^{2}$ Vanderbilt School of Medicine, Vanderbilt University Medical Center \\ * These authors contributed equally
}

Correspondence to: Jonathan Forbes at jonathan.forbes@vanderbilt.edu

URL: https://www.jove.com/video/2590

DOI: doi:10.3791/2590

Keywords: Medicine, Issue 53, microvascular, decompression, trigeminal, neuralgia, operation, video

Date Published: $7 / 5 / 2011$

Citation: Forbes, J., Cooper, C., Jermakowicz, W., Neimat, J., Konrad, P. Microvascular Decompression: Salient Surgical Principles and Technical Nuances. J. Vis. Exp. (53), e2590, doi:10.3791/2590 (2011).

\section{Abstract}

Trigeminal neuralgia is a disorder associated with severe episodes of lancinating pain in the distribution of the trigeminal nerve. Previous reports indicate that $80-90 \%$ of cases are related to compression of the trigeminal nerve by an adjacent vessel. The majority of patients with trigeminal neuralgia eventually require surgical management in order to achieve remission of symptoms. Surgical options for management include ablative procedures (e.g., radiosurgery, percutaneous radiofrequency lesioning, balloon compression, glycerol rhizolysis, etc.) and microvascular decompression. Ablative procedures fail to address the root cause of the disorder and are less effective at preventing recurrence of symptoms over the long term than microvascular decompression. However, microvascular decompression is inherently more invasive than ablative procedures and is associated with increased surgical risks. Previous studies have demonstrated a correlation between surgeon experience and patient outcome in microvascular decompression. In this series of 59 patients operated on by two neurosurgeons (JSN and PEK) since $2006,93 \%$ of patients demonstrated substantial improvement in their trigeminal neuralgia following the procedure-with follow-up ranging from 6 weeks to 2 years. Moreover, 41 of 66 patients (approximately 64\%) have been entirely pain-free following the operation.

In this publication, video format is utilized to review the microsurgical pathology of this disorder. Steps of the operative procedure are reviewed and salient principles and technical nuances useful in minimizing complications and maximizing efficacy are discussed.

\section{Video Link}

The video component of this article can be found at https://www.jove.com/video/2590/

Protocol

\section{Positioning}

1. Positioning is an integral component of microvascular decompression. Following induction of anesthesia, the patient is placed in pins while on the operating room table and moved to the lateral park bench position with side of the desired MVD placed up. Of note, JSN prefers to insert a lumbar drain prior to the procedure to allow greater control of intra-op drainage of CSF and cerebellar relaxation (the drain is then removed when the patient is discharged, often on POD2).

2. All pressure points are padded and an axillary roll is placed. The patient's chest and hips are taped securely to the table to allow rotation of the table, if necessary, later in the case. The patient's shoulder is taped down for purposes of visualization. The head is rotated approximately 10-15 degrees away from the affected side and the neck is flexed slightly so that the planned surgical trajectory is now almost orthogonal to the floor. The vertex is tilted slightly down. Special attention is made to ensure that there no pathologic compression of venous drainage from the head. Specifically, there should be room for at least two finger breadths between the mandible and upper thorax. Head rotation should not exceed 30 degrees.

3. c. The body is secured prior to three-point head fixation. Electrophysiologic monitoring (brainstem auditory evoked response and facial nerve monitoring) is then secured. Stealth navigation, if used, is registered.

\section{Incision}

1. Prior to prepping and draping, external landmarks are assessed. It is helpful to draw the inion-meatal line with an indelible marker. The iniomeatal line is drawn out first with an indelible marker. This dotted line represents the superficial representation of the location of the transverse sinus. Next, a dotted line is drawn overlying the course of the digastric groove. The portion of this line inferior to the iniomeatal line represents a superficial representation of the location of the sigmoid sinus. 
2. A curvilinear incision is drawn out approximately two fingerbreadths behind the pinna for the length of the ear. The patient is prepped and draped in the usual fashion.

3. Using a scalpel, the aforementioned incision is deepened. Dissection is carried down to the bone using a scalpel and monopolar cautery. Self-retaining retractors are placed.

\section{Craniotomy and dural opening}

1. With the superficial representation of the junction of the transverse and sigmoid sinuses in mind, drilling is initiated.

2. PEK prefers to drill the bone away to expose the transverse-sigmoid junction. A 3-cm craniectomy defect is usually created for access to the $\mathrm{CP}$ angle. This method avoids the possible risk posed by use of a drill with a footplate adjacent to the venous sinuses, potentially resulting in a dural sinus laceration.

3. JSN prefers to use a perforator drill for burr hole placement at the level of the transverse-sigmoid junction. Following this, a 3-cm craniotomy bone flap is created and set aside for replacement at the end of the case. The drill is then used to expose the remainder of the transverse and sigmoid sinuses. This method allows for replacement of the bone flap following the microvascular decompression.

4. Once the transverse and sigmoid sinuses have been exposed extradurally, the bone edges are waxed thoroughly. It is important to drill far enough laterally to allow proper dural reflection and visualization. When the mastoid air cells are encountered, they are vigorously waxed.

5. The dura is opened in a stellate fashion. The dural leaves are tacked up using 4-0 Nurolon and the dural incision is taken as close to the transverse-sigmoid junction as possible. This allows visualization of a corridor directly adjacent to the junction of the tentorium and petrous temporal bone.

\section{Exposure of the cerebellopontine angle}

1. After the dura has been reflected and sutured, a Leyla retractor is placed with telfa underlying the retractor. The cerebellum is elevated and gently retracted medially. Using suction and bipolar electrocautery, CSF is allowed to egress-resulting in gentle relaxation of the cerebellum.

2. With the cerebellum now having fallen away from the petro-tentorial junction, the retractor is advanced with further gentle elevation of the cerebellum. The superior petrosal vein complex is taken when necessary. When the petrosal vein is sacrificed, coagulation with bipolar cautery is followed by partially division with microscissors. The vessel is then further coagulated and then fully transected to ensure there is no hemorrhage following vessel ligation.

3. As "the corner is turned" and the cerebellopontine angle is exposed, the VII-VIII nerve complex is visualized first. During this exposure, vigilant attention is given to BAER (brainstem auditory evoked response) and facial nerve monitoring to guard against retraction injury. Superior and medial to the VII-VIII nerve complex, the trigeminal nerve is then visualized.

\section{Decompression of the trigeminal nerve}

1. Microscissors are used to open the arachnoid around the trigeminal nerve. Sharp dissection is necessary so that the nerve and superior cerebellar artery (SCA) can be inspected circumferentially. Inspection of the trigeminal nerve begins at the site of brainstem exit and proceeds laterally.

2. The most common vessel found in trigeminal neuralgia is a rostroventral loop of the SCA ${ }^{1}$. This vessel was the causal agent of compression in $53 \%$ of patients in this series.

3. After the causative vessel is identified, dissected, and freed, the vessel loop is mobilized away from the nerve. A small piece of Teflon is then placed underneath the vessel-elevating it off the trigeminal nerve.

4. Following decompression, the nerve is reexamined on all sides to be certain that no additional compressing vessels are present.

\section{Closure}

1. The wound is irrigated with saline and all retractors are removed. The dura is then closed using a running 4-0 Nurolon.

2. The remaining steps of closure differ slightly between attending. For JSN, the patch of the pericranium taken at the beginning of the case can be used to assist with water-tight closure of the dura. Multiple tacking holes are placed in the bone and 4-0 Nurolons are passed from the edge of the dura through the tacking holes and secured. Surgicel and Gelfoam are placed on top of the dura, and the bone flap is replaced with KLS screws and plates. Norian bone cement is then used to close off the remainder of the cranial opening in order to prevent later insertion of the muscle onto the dura. The wound is then copiously irrigated with Bacitracin irrigation and closed in layers with 2-0 Vicryls in multiple layers in the muscle, 3-0 Vicryls in the galea and dermis, and a running 4-0 Monocryl in the skin.

3. PEK prefers a slightly different method of closure. Following reapproximation of the dura, a piece of Gelfoam is placed overlying the dural incision. Methyl methacrylate is used to fill in the cranial defect and a small KLS plate used to secure the methylmethacrylate prosthetic to the skull. The skin and muscle is then closed as described above and covered with bacitracin ointment.

\section{Representative Results}

Using a review of CPT codes obtained from the VUMC Billing Department, the medical records of 59 patients who underwent microvascular decompression by one of two functional neurosurgeons (JSN or PEK) at Vanderbilt University Medical Center (VUMC) from the period of time from July 2006 to July 2009 were assessed in a retrospective fashion. Study data were collected and managed using REDCap electronic data capture tools2 (REDCap is a secure, web-based application based at Vanderbilt University designed to support data capture for research studies). A summary of the demographics of this patient population is visible in Table 1.

Regarding patient demographics (see Table 1), approximately $66 \%$ of patients were female. $10 \%$ had a history of multiple sclerosis and $19 \%$ had a history of previous surgical procedures for TGN (Table 2). Regarding operative results (see Table 3), 93\% of patients experienced significant improvement or full resolution of the facial pain at follow-up ranging from 6 weeks to 2 years. Our method of follow-up consisted of a 
retrospective review of the electronic medical record. $64 \%$ of patients reported themselves as pain-free at their most recent clinic visit following the procedure.

Approximately $15 \%$ of patients in the series suffered a CSF leak; $6(10 \%)$ of these patients required ventriculoperitoneal shunt insertion (VPSI). Of note, the rate of CSF leak was appreciably higher in the patient population prior to the routine use of bone flap or methylmethacrylate (MM) buttressing of closure. Specifically, $25 \%$ of patients who were closed without a bone flap or methylmethacrylate prosthetic flap developed a CSF leak. In contrast, only $8 \%$ of patients closed after bone or MM flap placement had evidence of post-operative CSF leakage. In addition to the results described above, Two (3\%) of patients suffered a wound infection. There were no instances of cerebellar damage. Two (3\%) of patients reported some degree of post-operative hearing loss.

\begin{tabular}{|l|l|}
\hline Patient Demographics & $20 / 39$ \\
\hline Gender (Male/Female) & $6 / 53$ \\
\hline History of Multiple Sclerosis (Yes/No) & $11 / 48$ \\
\hline History of previous surgical procedures for TGN (Yes/No) & \\
\hline
\end{tabular}

Table 1. Summary of patient demographics.

\section{Previous Procedures (11/59)}

\begin{tabular}{|l|l|l|}
\hline Type & Number & $\begin{array}{l}\text { Results: Pain free (PF) vs. Significantly } \\
\text { improved (SI) vs. No improvement (NI) }\end{array}$ \\
\hline Gamma knife & $5(8 \%)$ & $3 \mathrm{PF}, 2 \mathrm{SI}$ \\
\hline MVD & $2(3 \%)$ & $2 \mathrm{PF}$ \\
\hline RFL & $1(2 \%)$ & $\mathrm{PF}$ \\
\hline Multiple procedures & $2(3 \%)$ & $\mathrm{PF}, \mathrm{SI}$ \\
\hline
\end{tabular}

Table 2. Surgical outcomes of patients who underwent previous procedures prior to undergoing microvascular decompression (MVD) in the current series. Patients are stratified by the specific prior procedure. RFL = radiofrequency lesioning.

\begin{tabular}{|l|l|}
\hline Surgical Outcome and Complications & 4 months \\
\hline Average length of follow-up & $55(93 \%)$ \\
\hline Pain significantly improved or resolved entirely & $9(15 \%)$ \\
\hline CSF leak & $8(14 \%)$ \\
\hline Aseptic meningitis & $2(3 \%)$ \\
\hline Wound infection & $0(0 \%)$ \\
\hline Cerebellar damage & $2(3 \%)$ \\
\hline Hearing loss & \\
\hline
\end{tabular}

Table 3. Summary of surgical outcome and complications.

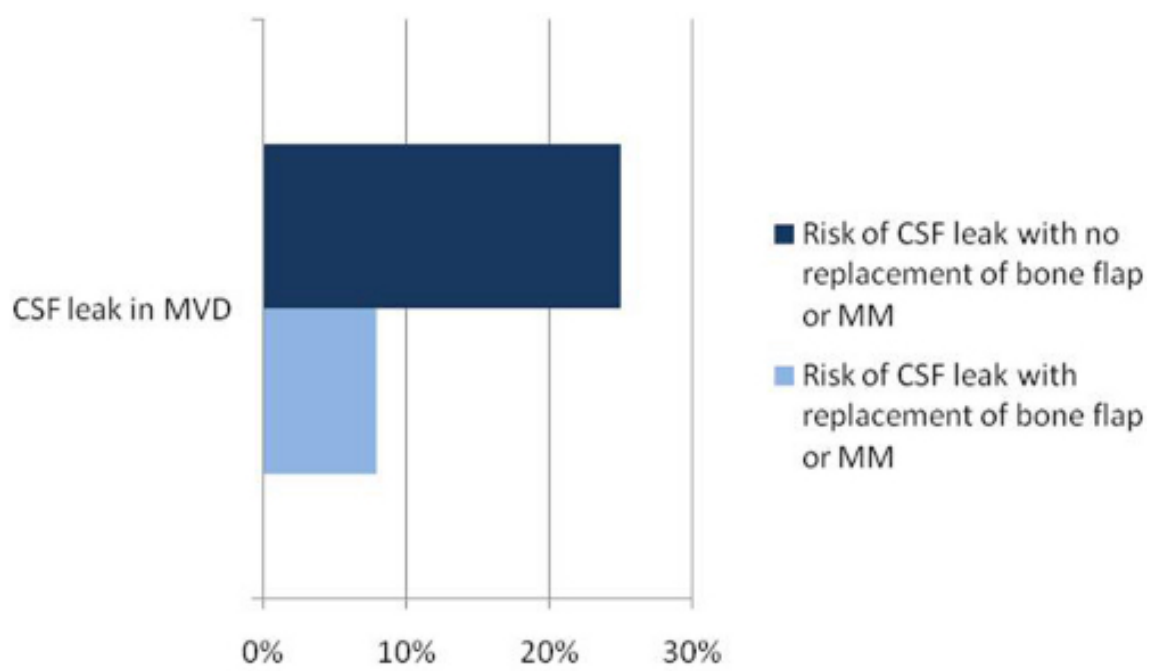

Figure 1. Operative results: rate of post-operative CSF leakage found to be heavily influenced by whether or not closure was buttressed with bone flap replacement or placement of methylmethacrylate (MM) flap. As a result, bone/MM flap replacement of craniectomy defect is now standard protocol for closure of these procedures. 


\title{
Post-operative Improvement in Pain
}

\author{
Pain mildly improved or not improved at all
}

Significant improvement in pain, although incomplete

Pain free

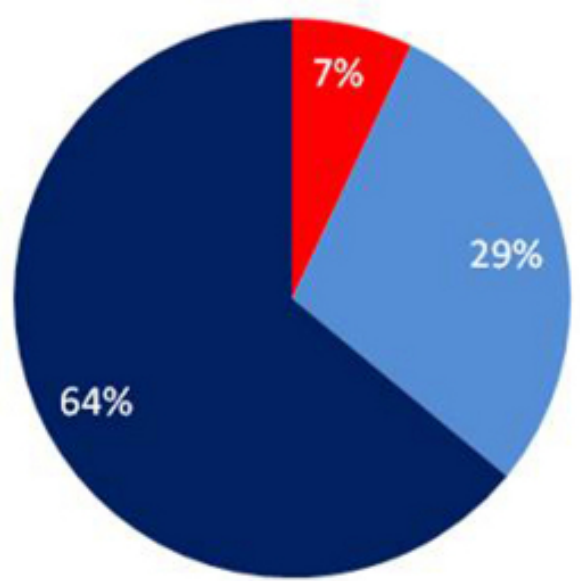

Figure 2. Stratification of results describing post-operative improvement in pain.

\section{Discussion}

Trigeminal neuralgia is a disorder associated with intense episodes of lancinating pain in the distribution of the trigeminal nerve. The severity of this pain can result in significant patient anxiety, malnutrition and even depression ${ }^{3}$. While possible proximal etiologies of this condition are diverse and include neoplasm or demyelination, $80-90 \%$ of cases are related to compression of the trigeminal nerve by an adjacent vessel ${ }^{4,5}$. In many cases of trigeminal neuralgia, pharmacological treatment is sufficient for adequate control of symptoms. However, the majority of patients require more definitive surgical measures6. Both microvascular decompression (MVD) and ablative procedures (e.g., radiosurgery, percutaneous radiofrequency lesioning, balloon compression, or glycerol rhizolysis) have been shown to be effective at controlling trigeminal neuralgia symptoms acutely-however, MVD has been demonstrated to be superior in preventing long-term recurrence ${ }^{7}$.

Since the first reported uses of MVD by Janetta in the 1960s, adaptations to the procedure have improved patient outcomes. The use of intraoperative auditory brainstem evoked potentials, in particular, has lessened surgical morbidity by monitoring for early irritation of the brainstem and cranial nerves and assisting in prevention of damage to these structures ${ }^{8}$. Recent reports estimate positive outcome rates of MVD for trigeminal neuralgia in the ranges of $77-94 \%{ }^{9,10,11}$. Nevertheless, significant postoperative complications, namely CSF leak, cerebellar damage and hearing loss ${ }^{9,10}$, still occur with the procedure. Additionally, recurrence of symptoms has been reported to occur in as many as $39 \%$ of patients ${ }^{10,11}$ following this procedure. In addition to being correlated with intraoperative variables such as nerve location, blood vessel, type and duration of symptoms ${ }^{6,7}$, the rate of recurrence also has been correlated with experience of the surgeon and hospital in performing MVD ${ }^{12}$. With proper technique, patient outcome can be maximized and adverse outcomes can be reduced.

This study examined the effectiveness of the surgical technique of MVD performed by two neurosurgeons at Vanderbilt University. The positive outcome rate we measured $(93 \%)$ is at the high range of those generally reported in the literature ${ }^{9,10}$. While average duration of follow-up in this series is small, patients are routinely not seen in clinic following the 6 -week visit if they remain pain-free and asymptomatic. Given that $64 \%$ of patients reported themselves to be entirely pain-free following the procedure, it is not surprising that the average duration of follow-up in this study is short. Present complication rates at our institution are comparable to those reported by others. Initially, MVDs were performed with a suboccipital craniectomy without replacement of the bone flap. After an internal review of the data, which demonstrated an elevated CSF leak of $25 \%$, the protocol was changed to include buttressing with either a methylmethacrylate flap or replacement of the bone flap prior to wound closure. Since this time, our CSF leak rate has approximated $8 \%$ - consistent with other rates $(2.4-12 \%)$ reported in the literature ${ }^{7,13}$. The percentage of our patients reporting hearing loss $(3 \%)$ also approximates figures reported in the literature $(0.8-2.8 \%)^{1,2,12,13},$. In no instance did we have a patient with evidence cerebellar damage.

Our experience suggests that monitoring with BAERs and bone flap replacement are worthwhile options in microvascular decompression. The salient surgical principles and technical nuances utilized at Vanderbilt University have been presented in video format. Furthermore, this video journal format uniquely presents the vascular pathology that many physicians do not routinely see outside the operating room. The results presented suggest that microvascular decompression can be a highly effective procedure performed with low patient morbidity. 


\section{Disclosures}

Dr. Peter Konrad and Dr. Joseph Neimat receive consulting fees and grant support from Medtronic, Inc. There is no associated conflict of interests with the data reported.

\section{References}

1. McLaughlin, M.R., Jannetta, P.J., Clyde, B.L., Subach, B.R., Comey, C.H., \& Resnick, D.K. Microvascular decompression of cranial nerves: lessons learned after 4400 operations. J Neurosurg. 90(1), 1-8 (1999).

2. Harris, P.A., Taylor, R., Thielke, R., Payne, J., Gonzalez, N., \& Conde, J.G. Research electronic data capture (REDCap): A metadata-driven methodology and workflow process for providing translational research informatics support. J Biomed Inform 42(2), 377-81 (2009).

3. Diener, H.C., Gendolla, A., \& Katsarava, Z. Diagnosis and treatment of head and facial pain. Internist (Berl) 49, 1343-1348 (2008).

4. Love, S., \& Coakham, H.B. Trigeminal neuralgia: Pathology and pathogenesis. Brain 124, 2347-2360 (2001).

5. Jannetta, P.J. Arterial compression of the trigeminal nerve at the pons in patients with trigeminal neuralgia. J. Neurosurg. 26, 159-162 (1967).

6. Barker, F.G., Jannetta, P.J., Bissonette, P.A.C., Larkins, M.V., \& Jho, H.D. The long-term outcome of microvascular decompression for trigeminal neuralgia. N.E.J.M. 334, 1077-1083 (1996).

7. Kabatas, S., Albayrak, S.B., Cansever, T., \& Hepgul, K.T. Microvascular decompression as a surgical management for trigeminal neuralgia: A critical review of the literature. Neurol India 57, 124-138 (2009).

8. Shin, J.C., Kim, Y.C., Park, C.I., \& Chung, U.H. Intraoperative monitoring of microvascular decompression in hemifacial spasm. Yonsei MJ. 37, 209-213 (1996).

9. Hitotsumatsu, T., Matsushima, T., \& Inoue, T. Microvascular decompression for treatment of trigeminal neuralgia, hemifacial spasm, and glossopharyngeal neuralgia: Three surgical approach variations. Neurosurg. 53, 1436-1443 (2003).

10. Sandell, T., \& Eide, P.K. Effect of microvascular decompression in trigeminal neuralgia patients with or without constant pain. Neurosurg. $\mathbf{6 3}$, 93-99 (2008).

11. Miller, J.P., Magill, S.T., Feridun, A., \& Burchiel, K.J. Predictors of long-term success after microvascular decompression for trigeminal neuralgia. J Neurosurg. 110, 620-626 (2009).

12. Kalkanis, S.N., Eskandar, E.N., Carter, B.S., \& Barker, F.G. Microvascular decompression surgery in the United States, 1996 to 2000 : Mortality rates, morbidity rates and the effects of hospital and surgeon volumes. Neurosurg. 52, 1251-1261 (2003).

13. Park, J.S., Kong, D.S., Lee, J.A., \& Park, K. Intraoperative management to prevent cerebrospinal fluid leakage after microvascular decompression surgery. Neurosurg Rev 30, 139-142 (2007). 